\title{
Kripke models for subtheories of CZF
}

\author{
Rosalie Iemhoff
}

Received: 16 November 2009 / Accepted: 19 November 2009 / Published online: 10 December 2009

C The Author(s) 2009. This article is published with open access at Springerlink.com

\begin{abstract}
In this paper a method to construct Kripke models for subtheories of constructive set theory is introduced that uses constructions from classical model theory such as constructible sets and generic extensions. Under the main construction all axioms except the collection axioms can be shown to hold in the constructed Kripke model. It is shown that by carefully choosing the classical models various instances of the collection axioms, such as exponentiation, can be forced to hold as well. The paper does not contain any deep results. It consists of first observations on the subject, and is meant to introduce some notions that could serve as a foundation for further research.
\end{abstract}

Keywords Kripke models · Constructive set theory · Constructible sets · Generic extensions · Intuitionistic logic

Mathematics Subject Classification (2000) $\quad$ 03F50 - 03F65 • 03C90

\section{Introduction}

Constructive set theory was introduced by John Myhill in 1975. It serves as a foundation for constructive mathematics, in much the same way that Zermelo-Fraenkel set theory serves as a foundation for classical mathematics. To obtain a constructive set theory, the first naive idea would be to only restrict the logic of classical set theory to intuitionistic logic but leave the axioms unchanged. This, however, does not work since already the Axiom of Foundation implies the law of the excluded middle. Therefore the set theoretic axioms have to be chosen with care. Some classical axioms are severely restricted, such as the Separation Axiom, that in a constructive setting allows

\footnotetext{
R. Iemhoff $(\varangle)$

Department of Philosophy, University Utrecht, Heidelberglaan 8, 3584 CS Utrecht, The Netherlands e-mail: Rosalie.Iemhoff@phil.uu.nl
} 
the construction of extensions based on bounded formulas only. For other axioms it suffices to replace them by a classically equivalent form. Set Induction, for example, is classically equivalent to Foundation and an accepted principle of constructive set theory.

Several systems have been proposed as a constructive set theory in the course of time, but we will concentrate in this paper on one particular brand of constructive set theory called Constructive Zermelo Fraenkel set theory CZF. CZF, which is based on Myhill's system, was introduced by Peter Aczel, who also confirmed the constructivity of the theory by providing an interpretation of it in Martin-Löf type theory [1-3,12]. Later Michael Rathjen constructed a realizability interpretation of the theory, and using this showed that CZF possesses constructive properties such as the disjunction property and various existence properties.

In this paper we study the Kripke models of Constructive Zermelo Fraenkel set theory. Kripke models are a useful tool to study constructive theories and because of their simplicity have been applied with great success to non-classical logics in general, and intuitionistic logic and Heyting Arithmetic in particular. Although nowadays there exist various models of CZF, such as the two interpretations discussed above, as well as others in topos theory [10] and in the form of Heyting algebras [5], Kripke models have been less investigated. In [7] Robert Lubarsky constructed two beautiful Kripke models of CZF that refute classical principles such as the Power Set Axiom.

In this paper the aim is to construct, given a frame, a model of CZF on that frame by using constructions from classical model theory such as the constructible sets and generic extensions. The idea is to attach classical models (the so-called local models) to the nodes of the frame and see how far, by carefully choosing the local models, the axioms of CZF can be forced to hold in the Kripke model.

This paper is just a small first step in that direction. We introduce the framework and provide requirements on the local models under which the model constructed on a given frame is indeed a Kripke model. Then we proceed to show that under certain natural conditions such as transitivity, the Kripke models thus constructed satisfy the axioms of CZF minus the collection axioms, although certain instances of the latter can be shown to hold as well. These results are straightforward: they follow easily from the definition of the models. The conditions on the local models for which this subtheory of CZF is satisfied in the final model are quite general, and there are many ways to construct Kripke models that satisfy the necessary requirements.

Finally we show how to produce Kripke models for various forms of collection by using specific properties of certain generic extensions. Given a frame we attach generic extensions of a certain ground model to the leaves of the frame, and the model itself to all interior nodes. We will see that several instances and variations of the collection axioms can be recovered via requirements on the partial orders on which the generic extensions are based, such as the countable chain condition. In particular, a bounded form of Exponentiation can be forced to hold.

These are modest results, but we do hope that the method presented in this paper, in particular the relation between properties of Kripke models and the generic extensions on which they are based, can be pushed further, and will ultimately lead to models of full CZF.

I thank an anonymous referee for useful and supportive comments. 


\section{$2 \mathrm{CZF}$}

Constructive Zermelo Fraenkel set theory CZF consists of the axioms and rules of intuitionistic logic with equality extended by the following axioms.

Extensionality $\quad a=b \leftrightarrow \forall x(x \in a \leftrightarrow x \in b)$.

Empty Set

$\exists x \forall y(y \notin x)$

Pairing

$\exists c \forall x(x \in c \leftrightarrow x=a \vee x=b)$.

Union

$\exists b \forall x(x \in b \leftrightarrow \exists y \in a(x \in y))$.

Bounded Separation $\quad \exists b \forall x(x \in b \leftrightarrow x \in a \wedge \varphi(x)) \quad$ ( $\varphi$ bounded).

Strong Infinity

$\exists \omega \forall x(x \in \omega \leftrightarrow x=\emptyset \vee \exists y \in \omega(x=y \cup\{y\}))$.

Set Induction

$\forall x(\forall y \in x \varphi(y) \rightarrow \varphi(x)) \rightarrow \forall x \varphi(x)$

Strong Collection

$\forall x \in a \exists y \varphi(x, y) \rightarrow$

$\exists b(\forall x \in a \exists y \in b \varphi(x, y) \wedge \forall y \in b \exists x \in a \varphi(x, y))$.

Subset Collection

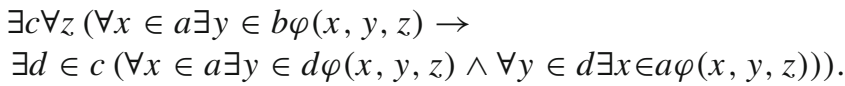

Bounded formulas are formulas in which every quantifier is bounded, thus of the form $\forall x \in y$ or $\exists x \in y . \mathrm{CZF}^{\mathrm{C}}$ is CZF minus the collection axioms, $\mathrm{CZF}^{\mathrm{ic}}$ is $\mathrm{CZF}^{\mathrm{C}}$ minus Set Induction.

Note that Set Induction is the constructive variant of Foundation, to which it is classically equivalent. It resembles the situation in Heyting Arithmetic, for which induction is one of the axioms, while the least number principle, which is classically equivalent to it, does not hold. Also note that Strong Collection is formulated in the way it is above since only full Separation would make it equivalent to the form most commonly used:

$$
\forall x \in a \exists y \varphi(x, y) \rightarrow \exists b \forall x \in a \exists y \in b \varphi(x, y) .
$$

Bounded Strong Collection is Strong Collection in which the $\varphi$ is a bounded formula. Below we will define what it means when a term is set-bounded in a formula. Given these notions, Set-bounded Subset Collection is Subset Collection in which the $\varphi(x, y, z)$ is a bounded formula in which $z$ is set-bounded.

The following two axioms are equivalent (over the other axioms) to, respectively, Bounded Separation and Subset Collection [4].

$$
\begin{array}{ll}
\text { Binary Intersection } & \exists c \forall x(x \in c \leftrightarrow x \in a \wedge x \in b) \\
\text { Fullness } & \exists c \forall r \in \operatorname{mv}\left({ }^{a} b\right) \exists r^{\prime} \in \operatorname{mv}\left({ }^{a} b\right)\left(r^{\prime} \subseteq r \wedge r^{\prime} \in c\right)
\end{array}
$$


In Fullness mv $\left({ }^{a} b\right)$ stands for all multi-valued relations from $a$ to $b$, i.e., all $r \subseteq a \times b$ such that $\forall x \in a \exists y \in b\langle a, b\rangle \in r$. The property $r^{\prime} \subseteq r$ expresses that $r^{\prime}$ is a refinement of $r$. Thus Fullness expresses that there is a set that contains at least one refinement for every $r \in \operatorname{mv}\left({ }^{a} b\right)$. Note that if $r: a \rightarrow b$, Fullness implies that $r \in c$. Therefore Fullness and Bounded Separation imply Exponentiation, that is, that $b^{a}$ is a set. This, however, does not imply that Power Set holds, because the existence of a set $\{0,1\}^{a}$ does not imply that the power set of $a$ exists: the existence of undecidable sets shows that not every subset of $a$ corresponds to a function in $\{0,1\}^{a}$.

We define

$$
\operatorname{Separation}_{\varphi} \quad \exists b \forall x(x \in b \leftrightarrow x \in a \wedge \varphi(x)),
$$

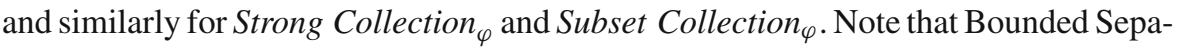
ration, Strong Collection and Subset Collection are axiom schemes, while their variants ()$_{\varphi}$ are formulas.

An axiom that is often used in constructive set theory is the Regularity Axiom which guarantees the existence of greatest fixed points, which are used in the setting of inductive definitions. In this paper the focus is on the other axioms and the Regularity Axiom will not be discussed any further here.

\section{Kripke models}

In this section we introduce the method to construct Kripke models for subtheories of CZF from classical models of ZF, which is the main object of study in this paper. Intuitively, given a frame $F$ and classical models $M_{i}$ for every node $i$ of $F$, the Kripke model will be the result of attaching model $M_{i}$ to node $i$, where the forcing of atomic formulas at a node corresponds to the validity of atomic formulas in the model at that node. Of course, in order to obtain a Kripke model various requirements have to be fulfilled. For example, for upwards persistency every model $M_{i}$ has to be a subset of the models at nodes above $i$, and every atomic formula that holds in $M_{i}$, should hold in all models at the nodes above $i$. The definitions below provide such restrictions. A collection of models is called sound for a given frame, it if satisfies all the necessary requirements.

Although in the applications to come classical models will be attached to nodes in a frame, one could also attach Kripke models to these nodes. The definitions below describe the construction on this level of generality, but it might be clarifying to keep in mind that in the theorems to come only the former restricted version of the construction is used. Clearly, the general case covers the specific case, as classical models are instances of Kripke models, namely Kripke models consisting of one node.

Note that in the general case we deal with nodes on two levels: nodes of the underlying frame $F$, and nodes in the Kripke models $M_{i}$ that we attach to the nodes of $F$. For a node $m$ in $M_{i}$ we denote forcing in $M_{i}$ at this node by $M_{i}, m \Vdash$, and in the final Kripke model we denote forcing at node $i$ in $F$ and node $m$ in $M_{i}$ by $\langle i, m\rangle \Vdash$. Here follow the details. 
Given a Kripke model $K$ we denote by $W^{K}, \preccurlyeq^{K}, D^{K}$ and $I^{K}$, respectively, its set of nodes, partial ordering, set of domains and its interpretations. A similar notation is used for frames. When $K$ is clear from the context we omit the superscript. Given a node $i$ in a model $K, D_{i}^{K}$ denotes the domain at node $i$, and $I_{i}^{K}$ the interpretation at node $i$.

Given a frame $F=(W, \preccurlyeq)$, we call a collection of (Kripke) models $\mathcal{M}=\left\{M_{i} \mid\right.$ $i \in W\}$ sound for $F$ if

$$
\begin{gathered}
\forall i, j \in W \forall m \in W^{M_{i}} \forall n \in W^{M_{j}}: i \preccurlyeq j \Rightarrow D_{m}^{M_{i}} \subseteq D_{n}^{M_{j}}, \\
\forall i, j \in W \forall m \in W^{M_{i}} \forall n \in W^{M_{j}} \forall a, b \in D_{m}^{M_{i}}: \\
i \preccurlyeq j \wedge M_{i}, m \Vdash P(a, b) \Rightarrow M_{j}, n \Vdash P(a, b) \quad(P \text { is }=\text { or } \in) .
\end{gathered}
$$

Given a frame $F$ and a collection of Kripke models $\mathcal{M}=\left\{M_{i} \mid i \in W^{F}\right\}$ that is sound for $F$, the Kripke model $K_{F}(\mathcal{M})=(W, \preccurlyeq, D, I)$ is defined as follows:

- $W \equiv_{\operatorname{def}} \bigcup\left\{\{i\} \times W^{M_{i}} \mid i \in W^{F}\right\}$,

- $D_{\langle i, m\rangle} \equiv_{\text {def }} D_{m}^{M_{i}}$,

- $\langle i, m\rangle \preccurlyeq\langle j, n\rangle \equiv_{\text {def }}\left(i=j \wedge m \preccurlyeq{ }^{M_{i}} n\right) \vee\left(i \prec{ }^{F} j\right)$,

- $I_{\langle i, m\rangle} \equiv{ }_{\text {def }} I_{m}^{M_{i}}$.

Thus $K_{F}(\mathcal{M})$ is obtained from $F$ by replacing node $i$ in $F$ by the Kripke model $M_{i}$. The models in $\mathcal{M}$ are called local models. We sometimes call $K_{F}(\mathcal{M})$ the final model.

Soundness guarantees the result to be a Kripke model:

Lemma 1 If $\mathcal{M}$ is sound for $F$, then in $K_{F}(\mathcal{M})$ :

$$
\langle i, m\rangle \preccurlyeq\langle j, n\rangle \Rightarrow(\langle i, m\rangle \Vdash \varphi \Rightarrow\langle j, n\rangle \Vdash \varphi) .
$$

In the following, when talking about a model $K_{F}(\mathcal{M})$, we tacitly assume that $\mathcal{M}$ is sound for $F$.

Clearly, classical models are models of the form $K_{F}(\mathcal{M})$, where $F$ consists of one node. We will mainly consider models of the form $K_{F}(\mathcal{M})$ for which $\mathcal{M}$ consists of classical models and $F$ is finite. Let us first consider an example of this form, where $F$ is the frame

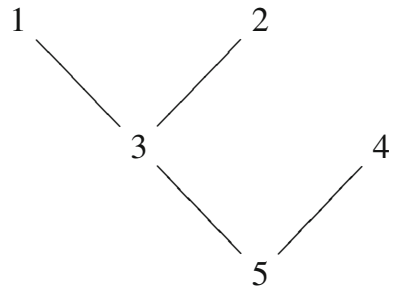


and $\mathcal{M}=\left\{M_{i} \mid i=1, \ldots, 5\right\}$ consists of classical models. Then $K_{F}(\mathcal{M})$ is the model

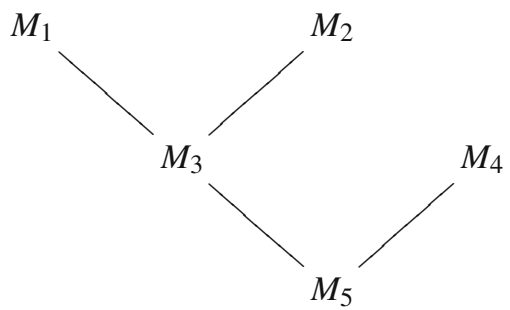

This model has 5 nodes.

An example for which the models in $\mathcal{M}$ consist of more than one node is for example given by the following frame $F$

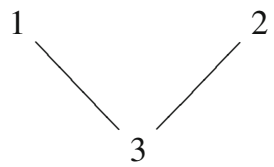

and the collection of models $\mathcal{M}=\left\{M_{i} \mid i=1,2,3\right\}$, where the frames of the models $M_{i}$ are of the form

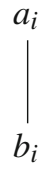

Thus $K_{F}(\mathcal{M})$ is the following 6 node model:

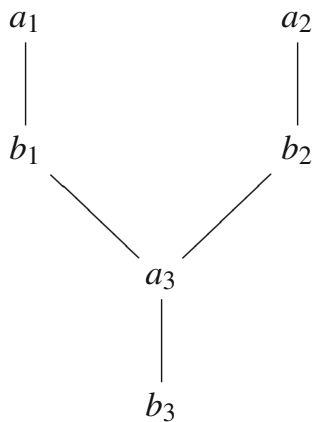

As mentioned above, if forcing in a model $M_{i}$ is considered this will always be indicated, as in expressions $M_{i}, m \Vdash \varphi$. In case the forcing is relative to $K_{F}(\mathcal{M})$, the model will be often omitted, which means that $\langle i, m\rangle \Vdash \varphi$ is short for $K_{F}(\mathcal{M}),\langle i, m\rangle \Vdash \varphi$ 
(recall that all nodes in $K_{F}(\mathcal{M})$ are of the form $\langle i, m\rangle$ ). Similar remarks apply to $\preccurlyeq^{M_{i}}$ and $\preccurlyeq K_{F}(\mathcal{M})$.

\subsection{Model properties}

This section discusses all the properties used to describe the requirements on $F$ and $\mathcal{M}$ under which $K_{F}(\mathcal{M})$ is a Kripke model of certain axioms of CZF. The properties are listed below, but the reader might consider to not consult them before the particular point at which they are used in the theorems, since their meaning will become clear from their application.

\section{D-formula}

Given a set $D, \varphi$ is a $D$-formula if all its parameters are in $D$.

\section{Set-bounded}

Given a formula $\varphi$ and a term $t, t$ is set-bounded in $\varphi$, if, when it occurs in $\varphi, \varphi \rightarrow t \in s$ is derivable in intuitionistic logic, for some term $s$ that occurs in $\varphi$. In $t \in s$, for example, $t$ is set-bounded while $s$ is not.

\section{Bounded}

A formula is bounded if all its quantified subformulas are of the form $\exists x \in a \varphi(x)$ or $\forall x \in a \varphi(x)$.

\section{$\in$-sound}

To establish that $K_{F}(\mathcal{M})$ satisfies certain axioms of CZF we will sometimes use that these axioms hold on the meta-level. For example, in the case of Set Induction we use that Set Induction holds in the real universe in which our objects live. For this we need the notion of $\in$-soundness.

$\mathcal{M}$ is $\in$-sound or sound for $\in$ if

$$
\forall a, b \in D_{m}^{M_{i}}: M_{i}, m \Vdash a \in b \Rightarrow a \in b .
$$

Here the $a \in b$ in the conclusion of $\Rightarrow$ refers to the underlying real universe, here taken to be classical set theory, if not explicitly stated otherwise. Note that classical $\in$-models are $\in$-sound.

\section{Transitive}

To force that certain simple axioms of CZF such as pairing and union hold in $K_{F}(\mathcal{M})$ we require that sets do not grow when moving up in the model, a property that is captured by the notion of transitivity. 
$\mathcal{M}$ is transitive at $\langle i, m\rangle$ if

$\forall\langle j, n\rangle \succcurlyeq\langle i, m\rangle \forall a \in D_{m}^{M_{i}} \forall b \in D_{n}^{M_{j}}: M_{j}, n \Vdash(b \in a \vee b=a) \Rightarrow b \in D_{m}^{M_{i}}$.

Note that this implies

$$
\forall\langle j, n\rangle \succcurlyeq\langle i, m\rangle \forall a \in D_{m}^{M_{i}} \forall b \in D_{n}^{M_{j}}:\langle j, n\rangle \Vdash(b \in a \vee b=a) \Rightarrow b \in D_{m}^{M_{i}} .
$$

$\mathcal{M}$ is transitive if it is transitive at every node. Note that if $\mathcal{M}$ consists of classical models, transitivity implies that the models are transitive in the usual sense, and vice versa.

To establish that $K_{F}(\mathcal{M})$ satisfies certain axioms of CZF we will often use that the local models satisfy these axioms. For example, in order to have $K_{F}(\mathcal{M})$ be a model of Bounded Separation, we need to understand for which formulas forcing at a node in a local model is equal to forcing at that node in $K_{F}(\mathcal{M})$. For in these cases $K_{F}(\mathcal{M})$ satisfies Bounded Separation if the local models do. The formulas for which we can establish such a property are the bounded formulas, for which we will show that they are preserved and decided in $K_{F}(\mathcal{M})$.

\section{Decidable}

A $D_{m}^{M_{i}}$-formula $\varphi(\bar{x})$ is decided at $\langle i, m\rangle$ if

$$
\forall \bar{a} \in D_{m}^{M_{i}} \forall\langle j, n\rangle \succcurlyeq\langle i, m\rangle:\langle j, n\rangle \Vdash \varphi(\bar{a}) \Leftrightarrow\langle i, m\rangle \Vdash \varphi(\bar{a}) .
$$

This is equivalent to $\langle i, m\rangle \Vdash \varphi(\bar{a}) \vee \neg \varphi(\bar{a}) . \varphi$ is decided in $K_{F}(\mathcal{M})$ if it is decided at every node $\langle j, n\rangle$ for which $\varphi$ is a $D_{n}^{M_{j}}$-formula. Observe that every formula is decided at the leaves of $K_{F}(\mathcal{M})$. $\mathcal{M}$ decides atomic formulas if all atomic formulas are decided in $K_{F}(\mathcal{M})$. Note that this means that for all $a, b \in D_{m}^{M_{i}} \cap D_{n}^{M_{j}}$ :

$$
M_{i}, m \Vdash a=b \Leftrightarrow M_{j}, n \Vdash a=b \quad M_{i}, m \Vdash a \in b \Leftrightarrow M_{j}, n \Vdash a \in b .
$$

Note that this notion depends on the models in $\mathcal{M}$ only.

\section{Preserved}

A $D_{m}^{M_{i}}$-formula $\varphi(\bar{x})$ is preserved at $\langle i, m\rangle$ if

$$
\forall \bar{a} \in D_{m}^{M_{i}}:\langle i, m\rangle \Vdash \varphi(\bar{a}) \Leftrightarrow M_{i}, m \Vdash \varphi(\bar{a}) .
$$

$\varphi$ is preserved in $K_{F}(\mathcal{M})$ if it is preserved at every node $\langle j, n\rangle$ for which $\varphi$ is a $D_{n}^{M_{j}}$-formula. Observe that every formula is preserved at the leaves of $K_{F}(\mathcal{M})$.

As we will see, in this paper we did not succeed in showing that, under the conditions considered in this paper, $K_{F}(\mathcal{M})$ is a model of the collection axioms. We do, however, recover certain instances of them. The requirements under which this holds 
are expressibility and collapse. These properties are rather strong, and we feel that in contrast to the results on the other axioms of CZF, the results using these two properties are unnatural, and leave ample room for improvement. In the last two sections we present some possible improvements by providing particular local models for which $K_{F}(\mathcal{M})$ is a model of a restricted form of Exponentiation and other principles related to functions.

\section{Expressible}

A $D_{m}^{M_{i}}$-formula $\varphi(x, y)$, in which $x, y$ are the only free variables, is expressible at $\langle i, m\rangle$ if

$\forall a, b \in D_{m}^{M_{i}}:\langle i, m\rangle \Vdash \forall x \in a \exists y \in b \varphi(x, y) \Leftrightarrow M_{i}, m \Vdash \forall x \in a \exists y \in b \psi_{m}^{i}(x, y)$.

The formulas $\psi_{m}^{i}$ are called the companions of $\varphi$. Observe that every $\varphi$ is a companion of itself at the leaves of $K_{F}(\mathcal{M})$. Corollary 1 below implies that every bounded $D_{m}^{M_{i}}$-formula is expressible at $\langle i, m\rangle$ by itself.

Lemma 2 If $\mathcal{M}$ is a transitive class of models of Pairing and decides atomic formulas, and $\varphi(x, y)$ is expressible by companions $\psi_{m}^{i}$, then

$$
\forall d, e \in D_{m}^{M_{i}}:\langle i, m\rangle \Vdash \varphi(d, e) \Leftrightarrow M_{i}, m \Vdash \psi_{m}^{i}(d, e) .
$$

Proof Consider $d, e \in D_{m}^{M_{i}}$. Because the $M_{i}$ are models of Pairing, there exists a set $\{d\} \in D_{m}^{M_{i}}$ such that

$$
M_{m}^{i} \Vdash \forall x(x \in\{d\} \leftrightarrow x=d) .
$$

The assumption that $\mathcal{M}$ is transitive and decides atomic formulas implies that also

$$
\langle i, m\rangle \Vdash \forall x(x \in\{d\} \leftrightarrow x=d) .
$$

This implies that

$$
\langle i, m\rangle \Vdash \varphi(d, e) \Leftrightarrow\langle i, m\rangle \Vdash \forall x \in\{d\} \exists y \in\{e\} \varphi(x, y) .
$$

The same reasoning gives

$$
M_{m}^{i} \Vdash \psi_{m}^{i}(d, e) \Leftrightarrow M_{m}^{i} \Vdash \forall x \in\{d\} \exists y \in\{e\} \psi_{m}^{i}(x, y) .
$$

Now we can use that $\varphi$ is expressible by $\psi_{m}^{i}$ at $\langle i, m\rangle$ to obtain the desired result. 


\section{Collapse}

Given $\langle j, n\rangle \succcurlyeq\langle i, m\rangle$, and a $D_{m}^{M_{i}}$-formula $\varphi(x, y, z)$, in which $x, y, z$ are the only free variables, then $\varphi(x, y, z)$ collapses from $\langle j, n\rangle$ to $\langle i, m\rangle$ if

$$
\begin{aligned}
& \forall c \in D_{n}^{M_{j}} \forall a, b \in D_{m}^{M_{i}}:\langle j, n\rangle \Vdash \forall x \in a \exists y \in b \varphi(x, y, c) \Rightarrow \\
& \left(\langle j, n\rangle \Vdash \neg \exists x \in a \text { or }\left(c \in D_{m}^{M_{i}} \text { and }\langle i, m\rangle \Vdash \forall x \in a \exists y \in b \varphi(x, y, c)\right)\right) .
\end{aligned}
$$

$\varphi(x, y, z)$ collapses if it collapses from $\langle j, n\rangle$ to $\langle i, m\rangle$ for all $\langle j, n\rangle \succcurlyeq\langle i, m\rangle$.

Lemma 3 If $\mathcal{M}$ is a transitive class of models of Set Induction that decides atomic formulas, and the $D_{m}^{M_{i}}$-formula $\varphi(x, y, z)$ is bounded, $z$ is set-bounded in it, and $x, y, z$ are the only free variables in it, then $\varphi(x, y, z)$ collapses.

Proof We have to show that for all $\langle j, n\rangle \succcurlyeq\langle i, m\rangle$ :

$$
\begin{aligned}
& \forall c \in D_{n}^{M_{j}} \forall a, b \in D_{m}^{M_{i}}:\langle j, n\rangle \Vdash \forall x \in a \exists y \in b \varphi(x, y, c) \Rightarrow \\
& \left(\langle j, n\rangle \Vdash \neg \exists x \in a \text { or }\left(c \in D_{m}^{M_{i}} \text { and }\langle i, m\rangle \Vdash \forall x \in a \exists y \in b \varphi(x, y, c)\right)\right) .
\end{aligned}
$$

Therefore suppose $\langle j, n\rangle \Vdash \forall x \in a \exists y \in b \varphi(x, y, c)$ and $\langle j, n\rangle \forall \neg \exists x \in a$. Thus for some $\langle h, k\rangle \succcurlyeq\langle j, n\rangle,\langle h, k\rangle \Vdash \exists x \in a \wedge \forall x \in a \exists y \in b \varphi(x, y, c)$. It follows that there exist elements $u, v$ such that $\langle h, k\rangle \Vdash u \in a \wedge v \in b \wedge \varphi(u, v, c)$. Hence $u, v \in D_{m}^{M_{i}}$ by transitivity. Since $z$ is set-bounded in $\varphi(x, y, z)$ it follows that $\langle h, k\rangle \Vdash c \in t$, for some term in $\varphi(u, v, c)$. Because Set Induction holds in the models, $t \neq c$. Since all terms in $\varphi(u, v, c)$ except $c$ belong to $D_{m}^{M_{i}}$, it follows that $c \in D_{m}^{M_{i}}$ by transitivity. That also $\langle i, m\rangle \Vdash \forall x \in a \exists y \in b \varphi(x, y, c)$ holds, follows from Corollary 1 below.

Recall that an $\in$-model is a model in which the membership relation is that of the universe.

Lemma 4 If $\mathcal{M}$ consists of classical transitive $\in$-models, then $\mathcal{M}$ is transitive, $\in$-sound and decides atomic formulas.

Note that in the lemma the requirement that the models of ZF are $\in$-models is not only needed for the $\in$-soundness, but also for the decidability of atomic formulas.

\subsection{Forcing in the two models}

Here follow some lemmas relating forcing in the local models to forcing in the final model. As we explained in the previous section, bounded formulas will be central in some of the theorems below. In this section we show that such formulas are preserved and decided in $K_{F}(\mathcal{M})$.

When we consider a formula at $M_{i}, m$ or $\langle i, m\rangle$ we tacitly assume that all its parameters belong to $D_{m}^{M_{i}}$, that is, that it is a $D_{m}^{M_{i}}$-formula. 
Lemma 5 If $\mathcal{M}$ is transitive and decides atomic formulas, then all bounded formulas are decided in $K_{F}(\mathcal{M})$.

Proof We have to show that for bounded formulas $\varphi$ :

$$
\forall\langle j, n\rangle \succcurlyeq\langle i, m\rangle:\langle i, m\rangle \Vdash \varphi \Leftrightarrow\langle j, n\rangle \Vdash \varphi .
$$

We use formula induction. For atomic formulas the lemma holds because $\mathcal{M}$ decides atomic formulas. That it holds for conjunction, disjunction and implication follows easily. We treat the existential quantifier, the universal quantifier being similar.

$\exists$ If $\langle j, n\rangle \Vdash \exists x \in a \psi(x)$, then for some $d \in D_{n}^{M_{j}},\langle j, n\rangle \Vdash d \in a \wedge \psi(d)$. By transitivity $d \in D_{m}^{M_{i}}$, and whence $\langle i, m\rangle \Vdash d \in a \wedge \psi(d)$ by the induction hypothesis. Thus $\langle i, m\rangle \Vdash \exists x \in a \psi(x)$.

Lemma 6 If $\mathcal{M}$ is transitive and decides atomic formulas, then all bounded formulas are preserved in $K_{F}(\mathcal{M})$.

Proof We have to show that for bounded $D_{m}^{M_{i}}$-formulas $\varphi$ :

$$
\langle i, m\rangle \Vdash \varphi \Leftrightarrow M_{i}, m \Vdash \varphi .
$$

We use formula induction. For atomic formulas the lemma holds because atomic formulas are preserved by definition of $K_{F}(\mathcal{M})$. That it holds for conjunction and disjunction follows easily. For implication we use the induction hypothesis and Lemma 5 that implies that all bounded formulas are decided in $K_{F}(\mathcal{M})$. We treat the universal quantifier, the existential quantifier being similar.

We have to show that

$$
\langle i, m\rangle \Vdash \forall x \in a \psi(x) \Leftrightarrow M_{i}, m \Vdash \forall x \in a \psi(x) .
$$

$\Rightarrow$ Suppose $n \succcurlyeq^{M_{i}} m$, and $d \in D_{n}^{M_{i}}$ and $M_{i}, n \Vdash d \in a$. Thus $d \in D_{m}^{M_{i}}$ by transitivity. Hence $\langle i, m\rangle \Vdash d \in a$ by decidability. Thus $\langle i, m\rangle \Vdash \psi(d)$. Hence $\langle i, n\rangle \Vdash \psi(d)$ by upwards persistency, and thus $M_{i}, n \Vdash \psi(d)$ by the induction hypothesis. This proves that $M_{i}, m \Vdash \forall x \in a \psi(x)$.

$\Leftarrow$ Suppose $\langle j, n\rangle \succcurlyeq\langle i, m\rangle$, and $d \in D_{n}^{M_{j}}$, and $\langle j, n\rangle \Vdash d \in a$. Hence $d \in D_{m}^{M_{i}}$ by transitivity and $M_{i}, m \Vdash d \in a$ by decidability. Thus $M_{i}, m \Vdash \psi(d)$. Hence $\langle i, m\rangle \Vdash \psi(d)$ by the induction hypothesis. Thus $\langle j, n\rangle \Vdash \psi(d)$. This proves that $\langle i, m\rangle \Vdash \forall x \in a \psi(x)$.

Observe that the condition of atomic decidability in the previous lemma cannot be replaced by atomic preservation, since the argument for implication in the proof by induction might no longer be true.

Corollary 1 If $\mathcal{M}$ is transitive and decides atomic formulas, then all bounded formulas are decided and preserved in $K_{F}(\mathcal{M})$.

Corollary 2 If $\mathcal{M}$ consists of transitive $\in$-models of ZF, then all bounded formulas are decided and preserved in $K_{F}(\mathcal{M})$. 


\section{Models of CZF}

\subsection{Models of CZF ${ }^{\text {ic }}$}

Proposition 1 If $\mathcal{M}$ is transitive and decides atomic formulas, then for all axioms $\varphi$ of $\mathrm{CZF}^{\mathrm{ic}}$ except Strong Infinity, if the models in $\mathcal{M}$ satisfy Extensionality and $\varphi$, then so does $K_{F}(\mathcal{M})$.

Proof The proof of this theorem is simple, but we have included all details for completeness' sake. Essential is that bounded formulas are preserved and decided.

Extensionality We have to show that

$$
\langle i, m\rangle \Vdash \forall x(x \in a \leftrightarrow x \in b) \leftrightarrow a=b .
$$

Observe that the formula is bounded. Since the $M_{i}$ are models of Extensionality, they satisfy this formula at all their nodes. Lemma 6 implies that whence the formula holds at all nodes in $K_{F}(\mathcal{M})$.

Empty Set Suppose the models in $\mathcal{M}$ are models of Empty Set. Thus for all nodes $m$ in $M_{i}$ there exists a set $\emptyset_{m}^{i} \in D_{m}^{M_{i}}$ that is the empty set at that node, i.e., such that

$$
M_{i}, m \Vdash \forall x\left(x \in \emptyset_{m}^{i} \rightarrow \perp\right) .
$$

Since $\forall x\left(x \in \emptyset_{m}^{i} \rightarrow \perp\right)$ is a bounded formula, Lemma 6 implies that it is forced at $\langle i, m\rangle$ too.

Pairing Suppose the models in $\mathcal{M}$ are models of Pairing. Thus there exist sets, denoted $\{a, b\}_{m}^{i}$, in $D_{m}^{M_{i}}$ for which

$$
M_{i}, m \Vdash \forall x\left(x \in\{a, b\}_{m}^{i} \leftrightarrow x=a \vee x=b\right) .
$$

Transitivity and the fact that atomic formulas are decided implies that $\forall x(x \in$ $\left.\{a, b\}_{m}^{i} \leftrightarrow x=a \vee x=b\right)$ is forced at $\langle i, m\rangle$ too.

Union Suppose the models in $\mathcal{M}$ are models of Union. The existence of sets $\cup a_{m}^{i} \in$ $D_{m}^{M_{i}}$ such that

$$
M_{i}, m \Vdash \forall x\left(x \in \cup a_{m}^{i} \leftrightarrow \exists y \in a(x \in y)\right),
$$

implies that

$$
\langle i, m\rangle \Vdash \forall x\left(x \in \cup a_{m}^{i} \leftrightarrow \exists y \in a(x \in y)\right)
$$

by transitivity and the fact that atomic formulas are decided.

Bounded Separation Suppose the models in $\mathcal{M}$ are models of Bounded Separation. Let $\varphi$ be a bounded formula. Thus there are sets $c_{m}^{i} \in D_{m}^{M_{i}}$ for which

$$
M_{i}, m \Vdash \forall x\left(x \in c_{m}^{i} \leftrightarrow x \in a \wedge \varphi(x)\right)
$$


Since $\forall x\left(x \in c_{m}^{i} \leftrightarrow x \in a \wedge \varphi(x)\right)$ is a bounded formula, it follows from Lemma 6 that $\langle i, m\rangle$ forces that formula too.

\subsection{Strong Infinity}

Proposition 2 If $\mathcal{M}$ is a transitive class of models of $\mathrm{CZF}^{\mathrm{ic}}$ that decides atomic formulas, then $K_{F}(\mathcal{M})$ is a model of Strong Infinity.

Proof Let $\emptyset_{m}^{i}$ be the sets such that

$$
M_{i}, m \Vdash \forall x \neg\left(x \in \emptyset_{m}^{i}\right) .
$$

Because the models are models of Strong Infinity, there are sets $\omega_{m}^{i}$ such that

$$
M_{i}, m \Vdash \forall x\left(x \in \omega_{m}^{i} \leftrightarrow x=\emptyset_{m}^{i} \vee \exists y \in \omega_{m}^{i}(x=y \cup\{y\})\right) .
$$

It suffices to show that $\langle i, m\rangle$ forces the formula, that is, that for $\langle j, n\rangle \succcurlyeq\langle i, m\rangle$ and $x \in D_{n}^{M_{j}}$ :

$$
\langle j, n\rangle \Vdash x \in \omega_{m}^{i} \Leftrightarrow\langle j, n\rangle \Vdash x=\emptyset_{m}^{i} \vee \exists y \in \omega_{m}^{i}(x=y \cup\{y\}) .
$$

We will use that for $x, y \in D_{m}^{M_{i}}$ :

$$
M_{i}, m \Vdash x=y \cup\{y\} \Leftrightarrow\langle i, m\rangle \Vdash x=y \cup\{y\} .
$$

It is not difficult to see that this holds, using transitivity, the decidability of atomic formulas, and that $x=y \cup\{y\}$ is equivalent to $\forall z(z \in x \leftrightarrow z \in y \vee z=y)$. $\Rightarrow$ Suppose $\langle j, n\rangle \Vdash x \in \omega_{m}^{i}$. We show that $\langle i, m\rangle$ forces $x=\emptyset_{m}^{i} \vee \exists y \in \omega_{m}^{i}(x=y \cup\{y\})$. Transitivity and the decidability of atomic formulas implies that $x \in D_{m}^{M_{i}}$ and $\langle i, m\rangle \Vdash$ $x \in \omega_{m}^{i}$. Thus $M_{i}, m$ forces $x \in \omega_{m}^{i}$, and whence $x=\emptyset_{m}^{i} \vee \exists y \in \omega_{m}^{i}(x=y \cup\{y\})$. The observations above imply that $\langle i, m\rangle$ forces that formula too.

$\Leftarrow$ First suppose $\langle j, n\rangle \Vdash x=\emptyset_{m}^{i}$. Note that $\langle i, m\rangle$ forces $\emptyset_{m}^{i} \in \omega_{m}^{i}$ since $M_{i}, m$ does. Hence $\langle j, n\rangle$ forces $x \in \omega_{m}^{i}$ by the equality axioms. Second, suppose for some $y,\langle j, n\rangle$ forces $y \in \omega_{m}^{i} \wedge x=y \cup\{y\}$. Thus $y \in D_{m}^{M_{i}}$ by transitivity, and $\langle i, m\rangle \Vdash y \in \omega_{m}^{i}$ by the decidability of atoms. There is a set $x^{\prime} \in D_{m}^{M_{i}}$ such that $\langle i, m\rangle$, and whence $M_{i}, m$, forces $x^{\prime}=y \cup\{y\}$. Thus $M_{i}, m$, and whence $\langle i, m\rangle$, forces $x^{\prime} \in \omega_{m}^{i}$. Hence $\langle j, n\rangle \Vdash x^{\prime} \in \omega_{m}^{i}$. Since $\langle j, n\rangle$ forces $x=x^{\prime}$ by Extensionality, it forces $x \in \omega_{m}^{i}$ by the equality axioms.

Corollary 3 If $\mathcal{M}$ is transitive and decides atomic formulas, and all models in $\mathcal{M}$ are models of $\mathrm{CZF}^{\mathrm{ic}}$, then $K_{F}(\mathcal{M})$ is a model of $\mathrm{CZF}^{\mathrm{ic}}$.

Combining this with Lemma 4 we obtain the following corollary.

Corollary 4 If $\mathcal{M}$ consists of transitive $\in$-models of $\mathrm{ZF}$, then $K_{F}(\mathcal{M})$ is a model of CZFic. 


\subsection{Models of Set Induction}

As mentioned in the beginning, the background theory in this paper is taken to be ZFC, but we do think that in most cases CZF would suffice. In this section we explicitly state in which background theory we work, because in the case of Set Induction it is instructive to see how the conditions on the local models have to be changed if we only allow constructive reasoning and wish the final model to be a model of Set Induction.

Proposition 3 (CZF) If $\mathcal{M}$ is sound for $\in$, then $K_{F}(\mathcal{M})$ is a model of Set Induction.

Proof Set Induction We show that $\forall x(\forall y \in x \varphi(y) \rightarrow \varphi(x)) \rightarrow \forall x \varphi(x)$ holds in $K_{F}(\mathcal{M})$. Note that it suffices to show that

$$
\langle i, m\rangle \Vdash \forall x(\forall y \in x \varphi(y) \rightarrow \varphi(x)) \Rightarrow\langle i, m\rangle \Vdash \forall x \varphi(x) .
$$

Therefore suppose $\langle i, m\rangle \Vdash \forall x(\forall y \in x \varphi(y) \rightarrow \varphi(x))$. We have to show that

$$
\forall\langle j, n\rangle \succcurlyeq\langle i, m\rangle \forall x \in D_{n}^{M_{j}}\langle j, n\rangle \Vdash \varphi(x) .
$$

Let

$$
\psi(x) \equiv_{\text {def }} \forall\langle j, n\rangle \succcurlyeq\langle i, m\rangle\left(x \in D_{n}^{M_{j}} \rightarrow\langle j, n\rangle \Vdash \varphi(x)\right) .
$$

It suffices to show that $\forall x(\forall y \in x \psi(y) \rightarrow \psi(x))$, since an application of Set Induction to this formula on the meta level then gives $\forall x \psi(x)$, i.e., $\langle i, m\rangle \Vdash \forall x \varphi(x)$. Thus assume $\forall y \in x \psi(y)$. To show $\psi(x)$, we prove that for $\langle j, n\rangle \succcurlyeq\langle i, m\rangle$ and $x \in D_{n}^{M_{j}}$, we have $\langle j, n\rangle \Vdash \forall y \in x \varphi(y)$. Since $\langle i, m\rangle$ forces $\forall x(\forall y \in x \varphi(y) \rightarrow \varphi(x))$, so does $\langle j, n\rangle$, and hence $\langle j, n\rangle \Vdash \varphi(x)$. This will show that $\psi(x)$, and completes the argument.

Therefore consider $\langle h, k\rangle \succcurlyeq\langle j, n\rangle, y \in D_{k}^{M_{h}}$ and $\langle h, k\rangle \Vdash y \in x$. Then $y \in x$ by $\in$-soundness. Because we have $\forall y \in x \psi(y)$, this implies $\langle h, k\rangle \Vdash \varphi(y)$. Thus indeed $\langle j, n\rangle \Vdash \forall y \in x \varphi(y)$.

By Lemma 4, Corollary 4, and the previous theorem we obtain the following corollary.

Corollary 5 If $\mathcal{M}$ consists of transitive $\in$-models of $\mathrm{ZF}$, then $K_{F}(\mathcal{M})$ is a model of $\mathrm{CZF}^{\mathrm{C}}$.

Proposition 4 (ZF) If $\mathcal{M}$ consists of models of Foundation, and $F$ is a frame without infinite ascending branches, then $K_{F}(\mathcal{M})$ is a model of Set Induction.

Proof Since all $M_{i}$ consist of one node, $\langle i, m\rangle$ becomes $i$, and we write $M_{j}$ for $D^{M_{j}}$. We have to show that

$$
i \Vdash \forall x(\forall y \in x \varphi(y) \rightarrow \varphi(x)) \Rightarrow i \Vdash \forall x \varphi(x) .
$$


Therefore suppose $i \Vdash \forall x(\forall y \in x \varphi(y) \rightarrow \varphi(x))$. Suppose $i \not \forall \forall x \varphi(x)$. Thus $i_{1} \forall \varphi\left(a_{1}\right)$ for some $a_{1} \in M_{i_{1}}$ and $i_{1} \succcurlyeq i$. This implies that $i_{1} \forall \forall x \in a_{1} \varphi(x)$, say $i_{2} \Downarrow \varphi\left(a_{2}\right)$ for some $a_{2} \in M_{i_{2}}$ such that $i_{2} \succcurlyeq i_{1}$ and $i_{2} \Vdash a_{2} \in a_{1}$. Hence $i_{3} \Vdash \varphi\left(a_{3}\right)$ for some $a_{3} \in M_{i_{3}}$ such that $i_{3} \succcurlyeq i_{2}$ and $i \Vdash a_{3} \in a_{2}$, etcetera. Since $F$ has no infinite ascending branches, there exists an $i_{j}$ such that for all $h \geq j$ with $h \in \omega, a_{h} \in M_{i_{j}}$ and $M_{i_{j}}=a_{h+1} \in a_{h}$. But this contradicts the fact that the models in $\mathcal{M}$ are models of Foundation.

\subsection{Models of Strong Collection}

The theorem below implies that under the standard conditions we have used so far, Bounded Collection holds in models of the form $K_{F}(\mathcal{M})$. Although many applications of collection require only Bounded Collection, we feel that the result in this section is rather weak. We chose to include it because the treatment of collection here is similar to the treatment of the other axioms we have encountered so far. In Section 6 several variations of this theorem will be proved showing that by a careful choice of the models in $\mathcal{M}$, other forms of collection can be forced to hold in $K_{F}(\mathcal{M})$.

Proposition 5 If $\mathcal{M}$ is transitive and decides atomic formulas, then for every $\varphi$ that is expressible by companions $\psi_{m}^{i}$, if the models in $\mathcal{M}$ are models of Strong Collection $\psi_{m}^{i}$ and Pairing, then $K_{F}(\mathcal{M})$ is a model of Strong Collection $_{\varphi}$.

Proof Let $\psi_{m}^{i}$ be the companions of $\varphi$. We show that for $a \in D_{m}^{M_{i}}$

$$
\langle i, m\rangle \Vdash \forall x \in a \exists y \varphi(x, y) \rightarrow \exists b(\forall x \in a \exists y \in b \varphi(x, y) \wedge \forall y \in b \exists x \in a \varphi(x, y)) .
$$

Therefore suppose $\langle i, m\rangle \Vdash \forall x \in a \exists y \varphi(x, y)$. We first show that

$$
M_{i}, m \Vdash \forall x \in a \exists y \psi_{m}^{i}(x, y) .
$$

Namely, if $n \succcurlyeq m$ and $x \in D_{n}^{M_{i}}$ such that $M_{i}, n \Vdash x \in a$, then $x \in D_{m}^{M_{i}}$ by transitivity, and $M_{i}, m \Vdash x \in a$ by decidability. Thus $\langle i, m\rangle \Vdash x \in a$ and whence $\langle i, m\rangle \Vdash \varphi(x, c)$ for some $c \in D_{m}^{M_{i}}$. Thus $M_{m}^{i} \Vdash \psi_{m}^{i}(x, c)$ by Lemma 2, and therefore $\exists y \psi_{m}^{i}(x, y)$, is forced at $M_{i}, m$, whence also at $M_{i}, n$.

Since the $M_{i}$ are models of Strong Collection $\psi_{m}^{i}$, this implies that there exist sets $b_{m}^{i} \in D_{m}^{M_{i}}$ such that

$$
M_{i}, m \Vdash \forall x \in a \exists y \in b_{m}^{i} \psi_{m}^{i}(x, y) \wedge \forall y \in b_{m}^{i} \exists x \in a \psi_{m}^{i}(x, y) .
$$

By the expressibility of $\varphi$ this implies

$$
\langle i, m\rangle \Vdash \forall x \in a \exists y \in b_{m}^{i} \varphi(x, y) \wedge \forall y \in b_{m}^{i} \exists x \in a \varphi(x, y) .
$$


Corollary 6 If $\mathcal{M}$ is transitive and decides atomic formulas, then if the models in $\mathcal{M}$ are models of Bounded Strong Collection, so is $K_{F}(\mathcal{M})$.

Proof If $\varphi$ is bounded, Corollary 1 implies that $\varphi$ is expressible by $\varphi$. Apply the previous theorem.

\subsection{Models of Subset Collection}

The theorem below implies that under the standard conditions we have used so far, Setbounded Subset Collection holds in models of the form $K_{F}(\mathcal{M})$. The same remark as in the case of Strong Collection applies here: the result is not strong since the expressive power of the formulas in the axiom is limited. In Section 7 we will encounter several variations of this theorem showing that under reasonable conditions a restricted version of Exponentiation holds in $K_{F}(\mathcal{M})$.

Proposition 6 If $\mathcal{M}$ is transitive and decides atomic formulas, then for every $\varphi$ that collapses and is expressible by formulas $\psi_{m}^{i}$, if the models in $\mathcal{M}$ are models of Subset Collection $_{\psi_{m}^{i}}$, then $K_{F}(\mathcal{M})$ is a model of Subset Collection $_{\varphi}$.

Proof We show that for $a, b \in D_{m}^{M_{i}}$,

$$
\begin{aligned}
& \langle i, m\rangle \Vdash \exists c \forall z[\forall x \in a \exists y \in b \varphi(x, y, z) \rightarrow \\
& \exists d \in c(\forall x \in a \exists y \in d \varphi(x, y, z) \wedge \forall y \in d \exists x \in a \varphi(x, y, z))] .
\end{aligned}
$$

Let $\psi_{m}^{i}$ be the companions of $\varphi$. Since the $M_{i}$ are models of Subset Collection $\psi_{m}^{i}$, there are $c_{m}^{i} \in D_{m}^{M_{i}}$ for which

$$
\begin{aligned}
& M_{i}, m \Vdash \forall z\left[\forall x \in a \exists y \in b \psi_{m}^{i}(x, y, z) \rightarrow\right. \\
& \left.\exists d \in c_{m}^{i}\left(\forall x \in a \exists y \in d \psi_{m}^{i}(x, y, z) \wedge \forall y \in d \exists x \in a \psi_{m}^{i}(x, y, z)\right)\right] .
\end{aligned}
$$

It suffices to show that for all $\langle j, n\rangle \succcurlyeq\langle i, m\rangle$ and all $z \in D_{n}^{M_{j}}$

$$
\begin{gathered}
\langle j, n\rangle \Vdash \forall x \in a \exists y \in b \varphi(x, y, z) \\
\Rightarrow \\
\langle j, n\rangle \Vdash \exists d \in c_{m}^{i}(\forall x \in a \exists y \in d \varphi(x, y, z) \wedge \forall y \in d \exists x \in a \varphi(x, y, z)) .
\end{gathered}
$$

Therefore assume $\langle j, n\rangle \succcurlyeq\langle i, m\rangle, z \in D_{n}^{M_{j}}$, and $\langle j, n\rangle \Vdash \forall x \in a \exists y \in b \varphi(x, y, z)$. Thus $\langle j, n\rangle \Vdash \neg \exists x \in a$ or $z \in D_{m}^{M_{i}}$ and $\langle i, m\rangle \Vdash \forall x \in a \exists y \in b \varphi(x, y, z)$. In the latter case, by expressibility $M_{i}, m \Vdash \forall x \in a \exists y \in b \psi_{m}^{i}(x, y, z)$. And thus for some $d \in D_{m}^{M_{i}}, M_{i}, m$ forces

$$
d \in c_{m}^{i} \wedge \forall x \in a \exists y \in d \psi_{m}^{i}(x, y, z) \wedge \forall y \in d \exists x \in a \psi_{m}^{i}(x, y, z) .
$$


By expressibility $\langle i, m\rangle$ forces

$$
d \in c_{m}^{i} \wedge \forall x \in a \exists y \in d \varphi(x, y, z) \wedge \forall y \in d \exists x \in a \varphi(x, y, z) .
$$

Thus so does $\langle j, n\rangle$, which is what we had to show. In the former case, $\langle j, n\rangle$ forces $\neg \exists x \in a$, it follows that $\langle i, m\rangle$, and whence $M_{i}, m$, forces $\Vdash \neg \exists x \in a$ too, by transitivity and decidability. Hence $M_{i}, m \Vdash \forall x \in a \exists y \in b \psi_{m}^{i}(x, y, z)$. Consider the $d$ for which (3). It follows that $M_{i}, m$, and whence $\langle i, m\rangle$, forces $\neg \exists x \in d$. Thus so does $\langle j, n\rangle$, which implies that $\langle j, n\rangle$ forces (4), which is what we had to show.

Corollary 7 If $\mathcal{M}$ is transitive and decides atomic formulas, then if the models in $\mathcal{M}$ are models of Set Induction and Set-bounded Subset Collection, $K_{F}(\mathcal{M})$ is a model of Set-bounded Subset Collection.

Proof Let $\varphi(x, y, z)$ be a bounded formula in which $z$ is set-bounded. By Corollary 1 , $\varphi$ is expressible by itself, and Lemma 3 implies that $\varphi$ collapses. Thus we can apply the previous theorem.

If we combine all results above we obtain the following corollaries.

Corollary 8 If $\mathcal{M}$ is sound for $\in$, consists of transitive models of $\mathrm{CZF}$, and decides atomic formulas, then $K_{F}(\mathcal{M})$ is a model of $\mathrm{CZF}^{\mathrm{C}}$ and Bounded Strong Collection and Set-bounded Subset Collection.

Corollary 9 If $\mathcal{M}$ decides atomic formulas and consists of transitive models of ZF, such that these are $\in$-models or $F$ has no infinite branches, then $K_{F}(\mathcal{M})$ is a model of $\mathrm{CZF}^{\mathrm{C}}$ plus Bounded Strong Collection and Set-bounded Subset Collection.

\section{Examples of models}

Here follow some examples of classes of models satisfying Corollary 9.

\subsection{Constructible set models}

A collection $\mathcal{M}$ of models sound for a frame $F$ is called an L-extension if every model that does not correspond to a leaf of $F$ is $\mathrm{L}$, that is, if all $M_{i}$, where $i$ is not a leaf of $F$, equal $\mathrm{L}$. In this case we denote $K_{F}(\mathcal{M})$ by $K_{F}^{\mathrm{L}}(\mathcal{M})$. The following is an example of such a model:

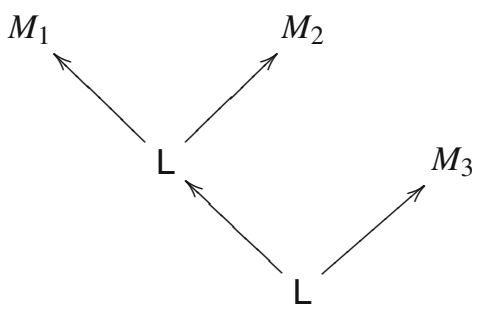


Recall that for every classical transitive proper class model $M$ of $\mathrm{ZF}, \mathrm{L}^{M}=\mathrm{L}$ (Theorem 3.5 in [6]), and thus $L$ is contained in $M$. Therefore a class of models $\mathcal{M}$ is an L-extension on a frame $F$ if the models in $\mathcal{M}$ corresponding to the leaves of $F$ are classical transitive proper class models of ZF, and all other models are $\mathrm{L}$.

Proposition 7 If $\mathcal{M}$ is an $\mathrm{L}$-extension of transitive $\in$-models of $\mathrm{ZF}$, then $K_{F}^{\mathrm{L}}(\mathcal{M})$ is a model of $\mathrm{CZF}^{\mathrm{C}}$, Bounded Strong Collection, and Set-bounded Subset Collection.

\subsection{Generic models}

A collection $\mathcal{M}$ of models that is sound for a frame $F$ is called an $M$-extension if $M_{i}$ is a generic extension $M\left[G_{i}\right]$ of $M$ if $i$ is a leaf of $F$, and it is the model $M$ otherwise. In this case we denote $K_{F}(\mathcal{M})$ by $K_{F}^{M}(\mathcal{M})$. Note that such models decide atomic formulas.

We follow the notation of the book by Kenneth Kunen on set theory [6]. We let $P_{i}$ be the partial order in $M$ with respect to which $G_{i}$ is generic. Since for every generic set $G$ every $m$ corresponds to a name $\breve{m}$, such that $(\breve{m})_{G}=m$, it follows that $M$ is a subset of every $M\left[G_{i}\right]$.

The following is an example of a model $K_{F}^{M}(\mathcal{M})$ :

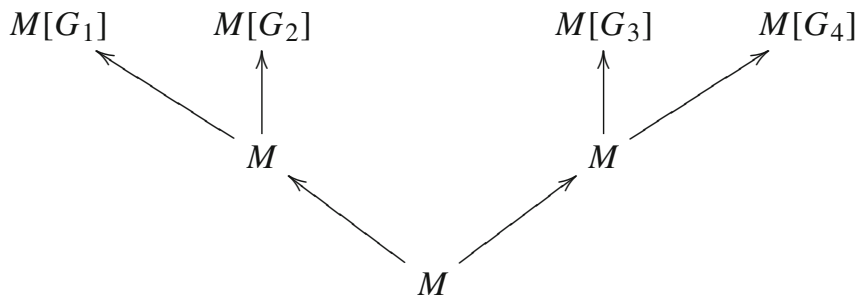

Proposition 8 If $\mathcal{M}$ is an $M$-extension of transitive models of ZF sound for a frame $F$ with no infinite ascending branches, then $K_{F}^{M}(\mathcal{M})$ is a model of $\mathrm{CZF}^{\mathrm{C}}$, Bounded Strong Collection, and Set-bounded Subset Collection.

\section{Functions}

The use of constructible sets and generic extensions in the results above does not seem to rely on constructibility or genericity in an essential way. The theorems merely serve as natural applications of the more abstract lemmas that were proved before. The results in this section, however, show that by carefully choosing generic models for the leaves of a frame, the final model can be forced to satisfy certain principles on the existence of functions at the interior nodes.

For cardinals $\kappa$ and natural numbers $n$ we define

$$
\begin{gathered}
\neg \neg \text { Functions }_{\kappa}^{n} \\
\text { for all } a \in M \text { with }|a|<\kappa: \neg \neg \forall x \in a \exists ! y \in b \varphi(x, y) \rightarrow \\
\exists f_{1}, \ldots, f_{n} \in b^{a} \forall x \in a \forall y \in b \neg \neg \bigvee_{i=1}^{n}\left(\varphi(x, y) \leftrightarrow f_{i}(x)=y\right) .
\end{gathered}
$$


The "for all $a$ with $|a|<\kappa$ " is meant externally, in the real universe. The $\neg \neg$ refers to the fact the antecedent of the implication is a double negated formula as well as to the fact that the $f_{i}$ are only not not equivalent to $\varphi$. The property states that the existence of certain functions at the leaves imply the existence, at the interior nodes, of a finite number of functions that at the leaves behave like the original functions.

Theorem 1 If $\kappa$ is a cardinal, $\mathcal{M}$ is an $M$-extension of transitive models of ZF, and $F$ is a finite frame with $n$ leaves, and all $P_{i}$ associated with the $M\left[G_{i}\right]$ are $\kappa$-closed, then $K_{F}^{M}(\mathcal{M})$ is a model of $\neg \neg$ Functions ${ }_{\kappa}^{n}$.

Proof Since the models in $\mathcal{M}$ are classical, we write $i$ instead of $\langle i, m\rangle$, and $M_{i}$ instead of $D^{M_{i}}$. Suppose $i \Vdash \neg \neg \forall x \in a \exists ! y \in b \varphi(x, y)$, where $a, b \in M_{i}$ and $|a|<\kappa$. Hence $j \Vdash \forall x \in a \exists ! y \in b \varphi(x)$ for all leaves $j \succcurlyeq i$. Since all formulas are preserved at leaves, it follows that $\varphi$ represents a function $f_{j}: a \rightarrow b$ that belongs to $M_{j}=M\left[G_{j}\right]$. If $i=j$ we are done. So suppose $i$ is not a leaf.

In Theorem VII.6.14 in [6] it is shown that if $P$ is $\kappa$-closed, then for all $G$ that are $P$-generic over $M$, for all $a, b \in M$ with $|a|<\kappa$, if $g: a \rightarrow b$ is in $M[G]$, then it is in $M$. Therefore $f_{j} \in M$. Hence $f_{j} \in b^{a}$ in $M$. That $\neg \neg \bigvee_{j=1}^{n}\left(\varphi(x, y) \leftrightarrow f_{j}(x)=y\right)$ follows easily.

$$
\begin{gathered}
{\text { Weak } \neg \neg \text { Functions }^{n}} \\
\neg \neg \forall x \in a \exists ! y \in b \varphi(x, y) \rightarrow \exists f_{1}, \ldots, f_{n} \in \mathcal{P}(b)^{a} \\
\forall x \in a \forall y \in b\left(\bigwedge_{i=1}^{n}\left|f_{i}(x)\right| \leq \omega \wedge \neg \neg \bigvee_{i=1}^{n}\left(\varphi(x, y) \rightarrow y \in f_{i}(x)\right)\right) .
\end{gathered}
$$

The Weakness refers to the fact that the $f_{i}$ belong to $\mathcal{P}(b)^{a}$ instead of $b^{a}$, but in contrast to $\neg \neg$ Functions ${ }_{\kappa}^{n}$, there is no restriction on the cardinality of $a$. Note that in the requirement $\left|f_{i}(x)\right| \leq \omega$ lies the non triviality of the property, as otherwise we could take $f_{i}(x)=b$, and the property would trivially hold.

Theorem 2 If $\mathcal{M}$ is an $M$-extension of transitive models of ZF sound for a finite frame $F$ with $n$ leaves, and $\left(P_{i} \text { has c.c.c. }\right)^{M}$, then $K_{F}^{M}(\mathcal{M})$ is a model of Weak $\neg \neg F$ unctions $^{n}$.

Proof Since the models in $\mathcal{M}$ are classical, we write $i$ instead of $\langle i, m\rangle$, and $M_{i}$ instead of $D^{M_{i}}$. Suppose $i \Vdash \neg \neg \forall x \in a \exists ! y \in b \varphi(x, y)$, where $a, b \in M_{i}$. Hence $j \Vdash \forall x \in a \exists ! y \in b \varphi(x)$ for all leaves $j \succcurlyeq i$. Since all formulas are preserved at leaves, it follows that $\varphi$ represents a function $g_{j}: a \rightarrow b$ that belongs to $M_{j}=M\left[G_{j}\right]$. If $i=j$ we can take $f_{j}(x)=\left\{g_{j}(x)\right\}$ and are done. So suppose $i$ is not a leaf.

In Lemma VII.5.5 in [6] it is shown that if ( $P$ has c.c.c.) ${ }^{M}$ and $g: a \rightarrow b$ is in $M[G]$ where $a, b \in M$, there is a map $f: a \rightarrow \mathcal{P}(b)$ in $M$ such that for all $x \in a$, $g(x) \in f(x)$ and $(|f(x)| \leq \omega)^{M}$.

Thus there exist $f_{j} \in M$ such that $f_{j}: a \rightarrow \mathcal{P}(b)$ and

$$
\forall x \in a\left(g_{j}(x) \in f_{j}(x)\right) \wedge\left(\left|f_{j}(x)\right| \leq \omega\right)^{M} .
$$


We have to show that the following formula is forced at $i$ :

$$
\forall x \in a \forall y \in b\left(\bigwedge_{j=1}^{n}\left|f_{j}(x)\right| \leq \omega \wedge \neg \neg \bigvee_{j=1}^{n}\left(\varphi(x, y) \rightarrow y \in f_{j}(x)\right)\right)
$$

The second part clearly holds as for leaves $j, j \Vdash \varphi(x, y) \leftrightarrow g_{j}(x)=y$. For the first part, note that since $\omega$ is absolute for transitive models, it belongs to all models in $\mathcal{M}$. Since $\bigwedge_{j=1}^{n}\left|f_{j}(x)\right| \leq \omega$ holds in $M$, there exist injections $h_{j}^{x}: f_{j}(x) \rightarrow \omega$ in $M$. It is easy to see that by Corollary $1, i$ forces that the $h_{j}^{x}$ are injections too, since injectivity can be expressed by a bounded formula.

\section{Exponentiation}

In this section we show that under certain conditions on the generic sets instances of exponentiation can be recovered. We write $f: a \rightarrow b$ as an abbreviation of " $f$ is a function from $a$ to $b$ ". Exponentiation is the axiom stating that the set $b^{a}$ of functions from $a$ to $b$ exists for all $a$ and $b$ :

$$
\text { Exponentiation } \forall a \forall b \exists c \forall f(f \in c \leftrightarrow f: a \rightarrow b) \text {. }
$$

For cardinals $\kappa$ we define a bounded version of Exponentiation:

\section{Exponentiation $_{\kappa}$}

$$
\text { for all } a \in M \text { with }|a|<\kappa: \forall b \exists c \forall f(f \in c \leftrightarrow f: a \rightarrow b)
$$

The "for all $a$ with $|a|<\kappa$ " is meant externally, in the real universe.

Theorem 3 If $\kappa$ is a cardinal, $\mathcal{M}$ is an $M$-extension of transitive models of ZF sound for a finite frame $F$ with $n$ leaves, and all $P_{i}$ associated with the $M\left[G_{i}\right]$ are $\kappa$-closed, then $K_{F}^{M}(\mathcal{M})$ is a model of Exponentiation . $_{\text {. }}$

Proof Since Exponentiation holds in the leaves of $K_{F}^{M}(\mathcal{M})$, it suffices to show that for all $i$ that are not leaves, for all $a, b \in M$ with $|a|<\kappa$, there is a set $c_{i} \in M$ such that $i$ forces $\forall f\left(f \in c_{i} \leftrightarrow f: a \rightarrow b\right)$. Take for all $c_{i}$ the set $b^{a}$ in $M$ consisting of all functions in $M$ from $a$ to $b$. This set exists because $M$ is a model of ZF, and thus of Exponentiation. We show that this $c$ satisfies the right property, that is, that for any node $i$ :

$$
i \Vdash \forall f(f \in c \leftrightarrow f: a \rightarrow b) .
$$

If $i$ is a leaf and $f \in c$, this means that $f \in M$ by the transitivity of $M$, and thus $f: a \rightarrow b$ holds in $M_{i}=M\left[G_{i}\right]$ too. If $f: a \rightarrow b$ in $M\left[G_{i}\right]$, then we use Theorem 6.14 in [6] stating that if $P$ is $\kappa$-closed, then for all $G$ that are $P$-generic over $M$, for all $a, b \in M$ with $|a|<\kappa$, if $g: a \rightarrow b$ is in $M[G]$, then it is in $M$. Thus $f \in M$, and whence $f \in c$. 
If $i$ is not a leaf, we only have to consider the case that $f \in M$, as $f \in M\left[G_{i}\right]$ has been treated above. But in this case $f \in c \leftrightarrow f: a \rightarrow b$ is clearly forced at $i$.

The results in the last two sections show how the choice of the generic sets can force the final Kripke model to be a model of certain instances of collection. The obvious open problem is whether this method can be pushed further to obtain models of larger subtheories of CZF than the theories treated in this paper.

Open Access This article is distributed under the terms of the Creative Commons Attribution Noncommercial License which permits any noncommercial use, distribution, and reproduction in any medium, provided the original author(s) and source are credited.

\section{References}

1. Aczel, P. : The type-theoretic interpretation of constructive set theory. In: Troelstra, A.S., van Dalen, D. (eds.) Logic Colloquium 1977, pp. 55-66. North-Holland, Amsterdam (1978)

2. Aczel, P. : The type-theoretic interpretation of constructive set theory: choice principles. In: Macintyre, A. (ed.) The L.E.J. Brouwer Centenary Symposium, pp. 1-40. Amsterdam (1982)

3. Aczel, P. : The type-theoretic interpretation of constructive set theory: inductive definitions. In: Marcus, R.B. (ed.) Logic, Methodology and Philosophy of Science VII, pp. 17-49. North-Holland, Amsterdam (1986)

4. Aczel, P., Rathjen, M.: Notes on constructive set theory, Manuscript (2007)

5. Gambino, N.: Heyting-valued interpretations for constructive set theory. Ann. Pure Appl. Logic 137(13), 164-188 (2006)

6. Kunen, K.: Set Theory-An Introduction to Independence Proofs, Studies in Logic and the Foundations of Mathematics. Elsevier, Amsterdam (1980)

7. Lubarsky, R.: Independence results around constructive ZF. Ann. Pure Appl. Logic 132(2-3), 209225 (2005)

8. Lubarsky, R.: CZF and second order arithmetic. Ann. Pure Appl. Logic 141(1-2), 29-34 (2006)

9. Myhill, J.: Constructive set theory. J. Symbolic Logic 40, 347-382 (1975)

10. van Oosten, J.: Realizability: An Introduction to its Categorical Side. Elsevier, Amsterdam (2008)

11. Rathjen, M.: The disjunction and related properties for constructive Zermelo-Fraenkel set theory. J. Symbolic Logic 70(4), 1233-1254 (2005)

12. Rathjen, M., Tupailo, S.: Characterizing the interpretation of set theory in Martin-Löf type theory. Ann. Pure Appl. Logic 141(3), 442-471 (2006) 